\title{
Spirulina platensis Alleviates the Liver, Brain and Heart Oxidative Stress in Type 1 Diabetic Rats
}

\author{
Francisca Adilfa de Oliveira Garcia ${ }^{1,2}{ }^{*}$, Violet G. Yuen' ${ }^{1}$, Helioswilton Sales de Campos ${ }^{3}$, \\ Eveline Turatti ${ }^{4}$, Glauce Socorro de Barros Viana ${ }^{2,5}$, Carlo José Freire Oliveira ${ }^{3}$, John H. McNeill ${ }^{1}$
}

${ }^{1}$ Faculty of Pharmaceutical Sciences, The University of British Columbia (UBC), Vancouver, Canada

${ }^{2}$ Departamento de Fisiologia, Faculdade de Medicina Estacio de Juazeiro do Norte (ESTACIO), Juazeiro do Norte, Brazil

${ }^{3}$ Departamento de Microbiologia, Imunologia e parasitologia, Universidade Federal do Triangulo Mineiro (UFTM), Minas Gerais, Brazil

${ }^{4}$ Faculdade de Odontologia, Universidade de Fortaleza (UNIFOR), Fortaleza, Brazil

${ }^{5}$ Departamento de Farmacologia, Universidade Federal do Ceará(UFC), Fortaleza, Brazil

Email: *adilfabio@hotmail.com

How to cite this paper: de Oliveira Garcia, F.A., Yuen, V.G., de Campos, H.S., Turatti, E., de Barros Viana, G.S., Oliveira, C.J.F. and McNeill, J.H. (2018) Spirulina platensis Alleviates the Liver, Brain and Heart Oxidative Stress in Type 1 Diabetic Rats. Food and Nutrition Sciences, 9, 735-750.

https://doi.org/10.4236/fns.2018.96056

Received: May 19, 2018

Accepted: June 25, 2018

Published: June 28, 2018

Copyright $\odot 2018$ by authors and Scientific Research Publishing Inc. This work is licensed under the Creative Commons Attribution International License (CC BY 4.0).

http://creativecommons.org/licenses/by/4.0/

\begin{abstract}
Spirulina platensis (SPI) is a microalga with a high content of functional compounds, such as phenolics, phycocyanins and polysaccharides that has been shown to have antioxidant, anti-inflammatory, hypoglycemic, neuroprotective and immunomodulatory effects. The objectives of the present work were to study the possible effects of SPI treatment on the glycemic-lipid profile, oxidative stress, lipid peroxidation and cardiac performance in diabetic rats. Diabetes was induced by streptozotocin (STZ) in male Wistar rats. In diabetic animals SPI, at a dose of $50 \mathrm{mg} / \mathrm{kg} /$ day, reduced lipid peroxidation, nitrite levels and lipids in plasma and tissues. SPI exhibited an effective improvement on $+\mathrm{dP} / \mathrm{dT}$ and $-\mathrm{dP} / \mathrm{dT}$ in non-diabetic rats. This study showed that SPI significantly suppressed nitrite generation and lipoperoxidation in the hearts of diabetic animals, as well as an improvement in the cardiac function in control SPI-treated rats which is consistent with several studies that demonstrated the protective effect of antioxidants on oxidative stress-mediated injury caused by reactive oxygen species (ROS) produced in diabetic myocardial tissues.
\end{abstract}

\section{Keywords}

Spirulina platensis, Lipid Profile, Cardiac Function, Oxidative Stress, Diabetes

\section{Introduction}

Spirulina platensis is a multicellular and filamentous edible blue-green alga. Due 
to abnormally high levels of chlorophyll in SPI, it was initially placed in the plant kingdom but was later shifted to the bacterial kingdom based on new understanding of SPI physiology, genetics and biochemical properties. SPI cells form long strands which look similar to a coiled spring; thus the name SPI, meaning "little spring" [1]. SPI is found naturally in alkaline lakes. It has also been cultured in a controlled environment for human consumption [1] [2].

SPI is used as a food supplement and the nutritional and therapeutic values have been well documented [2]. SPI contains $62 \%$ amino acids, is the world's richest natural source of vitamin B12 and contains a whole spectrum of natural mixed carotenes and xanthophyll phytopigments. SPI is wrapped with a soft cell wall formed from complex sugars and proteins like rhamnose, xylose, glucose, galactose, and arabopyranose glucuronic acid [3] [4]. Algae of the genus Spirulina spp present approximately 15\% of biliproteins (C-phycocyanin, allophycocyanin and phycoerythrin) [4], with C-phycocyanin being the major protein component of SPI.

More recently SPI has been found to have additional pharmacological properties through a variety of active constituents. SPI exhibits antioxidant, anti-inflammatory, neuroprotective, immunomodulatory, antihyperlipidemic, cardiovascular and anti-diabetic properties, making it a potential drug candidate in the therapeutic management of chronic disorders such as diabetes and hypertension [4]-[9] [2] [25]-[32] [44] [45].

Diabetes is a metabolic disorder characterised by chronic hyperglycaemia. The long-term effects of diabetes mellitus include cellular injury, inflammation and failure of various organs [10].

Diabetes mellitus is a well-known risk factor for the development of heart failure independent of coronary heart disease and hypertension and may cause a cardiomyopathy [11] [12] [13]. A significant number of diabetic patients exhibit diabetic cardiomyopathy (DCM), however, the development of DCM remains poorly understood and the underlying mechanisms have not yet been clearly elucidated. For many authors DCM is characterized by sustained hyperglycaemia and hyperlipidemia, oxidative stress, defective calcium handling, apoptotic and necrotic cell death, altered mitochondrial function, inflammation and myocardial fibrosis [11].

Clinical studies have showed that DCM increases the risk of death in the patients [12]. Since there are no effective approaches to preventing the development and progression of diabetic cardiomyopathy in the clinic, the search for new therapeutic targets for the prevention or protection from DCM would be an important in therapeutic development [13].

An important clue to the prevention of DCM is the understanding that oxidative stress plays an important role in the development of diabetes and diabetic complications. Hyperglycemia-induced activation of several pathways results in the excessive formation of reactive oxygen species (ROS) that is toxic to the cell. The ROS interaction with lipids produce lipid peroxidation product like malon- 
dialdehyde (MDA) which further damages the cells, the interaction of nitric oxide (NO) with ROS causes the production of several reactive nitrogen species (RNS ) such as nitrogen dioxide $\left(\mathrm{NO}_{2}^{-}\right)$, peroxynitrite (OONO-), dinitrogen trioxide $\left(\mathrm{N}_{2} \mathrm{O}_{3}\right)$ and nitrous acid $\left(\mathrm{HNO}_{2}\right)$ that potentiate cellular damage [14] [15]. Also, the variation in the levels of antioxidant enzymes, such as superoxide dismutase (SOD) and glutathione peroxidase (GSH-Px), tissue susceptible to oxidative stress leading to the development of diabetic complications such as DCM [15].

It is well recognized that stress is closely associated with the pathogenesis of diabetes, and long-term exposure to oxidative stress in diabetes leads to chronic inflammation [16] [17]. It has been suggested that the ideal therapeutic agent to manage the multifactorial aspects of diabetes should act concomitantly, in the modulation of the molecular signaling where oxidative stress and inflammatory responses have been shown to cross-talk and closely interact with each other [16].

Previous studies in our partner laboratory in Brazil focused on investigating the anti-inflammatory effects of SPI and showed that the oral administration of SPI for 5 days, at doses of 50 and $100 \mathrm{mg} / \mathrm{kg} /$ day significantly decreased paw edema volume in alloxan-induced diabetic mice and rats. The anti-inflammatory effect of SPI was further confirmed by a decrease in TNFa immunostaining in the inflamed paw and in the myeloperoxidase release from human neutrophils [5].

The objectives of the present work were to study the possible effects of SPI treatment on the glycemic-lipid profile, oxidative stress, lipid peroxidation and cardiac performance in STZ-induced diabetic rats.

\section{Materials and Methods}

\subsection{Cultivation and Collection of Botanical Material}

The cultivation of SPI needs intense sunlight, high temperature and low rains, besides nutrients and a $\mathrm{pH}$ between 9 and 10. Initially, the innocula received from Antenna (Geneve, Switzerland) were cultivated in our laboratory of Federal University of Ceara, in Brazil, utilizing Zarrouk media [18]. After filtration, the material was weighed to determine the wet biomass, and submitted to desiccation for $5 \mathrm{~h}\left(50^{\circ} \mathrm{C}\right)$. The dried material was weighed to determine the dried biomass and the production per square meter $\left(8\right.$ to $\left.10 \mathrm{~g} / \mathrm{day} / \mathrm{m}^{2}\right)$.

\subsection{Experimental Diabetes}

\section{Animals and Induction of Diabetes}

Male Wistar rats weighing between 200 and $250 \mathrm{~g}$ were obtained from Charles River Laboratories Inc., Laval, Quebec and allowed to acclimate in the local vivarium. The rats were housed on 12-hour light-dark cycle and were allowed free access to standard laboratory diet (Purina rat chow) and drinking water in the Modified Barrier Facility at the University of British Columbia (UBC), Vancou- 
ver, British Columbia, Canada. Care was given in accordance with the principles of the Canadian Council on Animal Care. The experimental protocol used in this study was reviewed and approved by the Animal Care Committee of the University of British Columbia. Diabetes was induced by a single intravenous tail vein injection (under halothane anaesthesia) of streptozotocin (STZ) (Sigma, St. Louis, MO. USA) $(60 \mathrm{mg} / \mathrm{kg})$. Immediately before use STZ was freshly dissolved in $0.9 \%$ saline at a concentration of $60 \mathrm{mg} / \mathrm{ml}$. An equivalent volume of saline was administrated to control animals. The rats were considered diabetic if blood glucose levels were greater than or equal to $15 \mathrm{mM} 72$ hours after STZ injection. The rats were divided into 4 treatment groups ( $\mathrm{n}=8$ each): control (C), control plus SPI (CT), diabetic (D) and diabetic plus SPI (DT). Dried Spirulina prepared in distilled water was administered by oral gavage daily at a dose of 50 $\mathrm{mg} / \mathrm{kg}$ for 6 weeks.

\section{1) Isolated Working Heart Procedure}

At termination animals were anaesthetized with a subcutaneous injection of pentobarbital, the chest cavity opened, the heart excised and the cardiac function was determined by the isolated working heart methodology used extensively in this laboratory [19] [20].

\section{2) Biochemical Parameters}

Plasma and tissues (liver, brain and heart) were collected, snap-frozen in liquid nitrogen, and stored at $-80^{\circ} \mathrm{C}$ for biochemical measurements. Plasma glucose was analyzed using a glucose analyzer II (Beckman instruments, USA). Plasma cholesterol and triglycerides levels, tissue malondialdehyde (MDA), nitrite, superoxide dismutase (SOD) levels were analyzed using test kits from Cayman Chemical Co. (Ann Arbor, MI).

\section{3) Effects of SPI on the Hepatic Tissue Morphology}

At termination tissue samples of liver were promptly excised, rinsed with normal saline and fixed into $10 \%$ neutral formalin for histopathological examinations, by standard hematoxylin \& eosin staining and examined under light microscope (Olympus BX51, Tokyo, Japan).

\section{4) Statistical Analysis}

Data were expressed as mean \pm standard error of mean (SEM). Statistical analyses were done with either one-way ANOVA or GLM repeated measures ANOVA as described in the figure legend followed by a post-hoc Newman-Keuls multiple comparisons test. Graph Pad prism program (version 7) and NCSS were used as analyzing software. A level of $\mathrm{p} \leq 0.05$ was taken as significant. The number of animals per group per study is as described in the figure legend.

\section{Results}

\subsection{SPI $50 \mathrm{mg} / \mathrm{kg} /$ day Reduced Plasma Lipid Levels in Diabetic Animals and Reduced Body Weight in Control Treated Animals}

Plasma glucose, cholesterol, triglycerides were measured in control and diabetic rats at termination. Plasma glucose levels were significantly higher in diabetic 
groups as compared to control. SPI, at a daily dose of $50 \mathrm{mg} / \mathrm{kg} /$ day, was not effective in reducing hyperglycemia during 6 weeks of treatment (Figure 1(a)).

SPI $50 \mathrm{mg} / \mathrm{kg} /$ day decreased significantly the levels of plasma lipids. After 6 weeks of SPI treatment, plasma triglyceride levels were restored to normal levels in diabetic treated animals (Figure 1(b), Figure 1(c)).

SPI $50 \mathrm{mg} / \mathrm{kg} /$ day reduced body weight in control treated animals from week 3 of treatment through to termination. There was no effect of treatment on body weight in the diabetic group (Figure 1(d)).
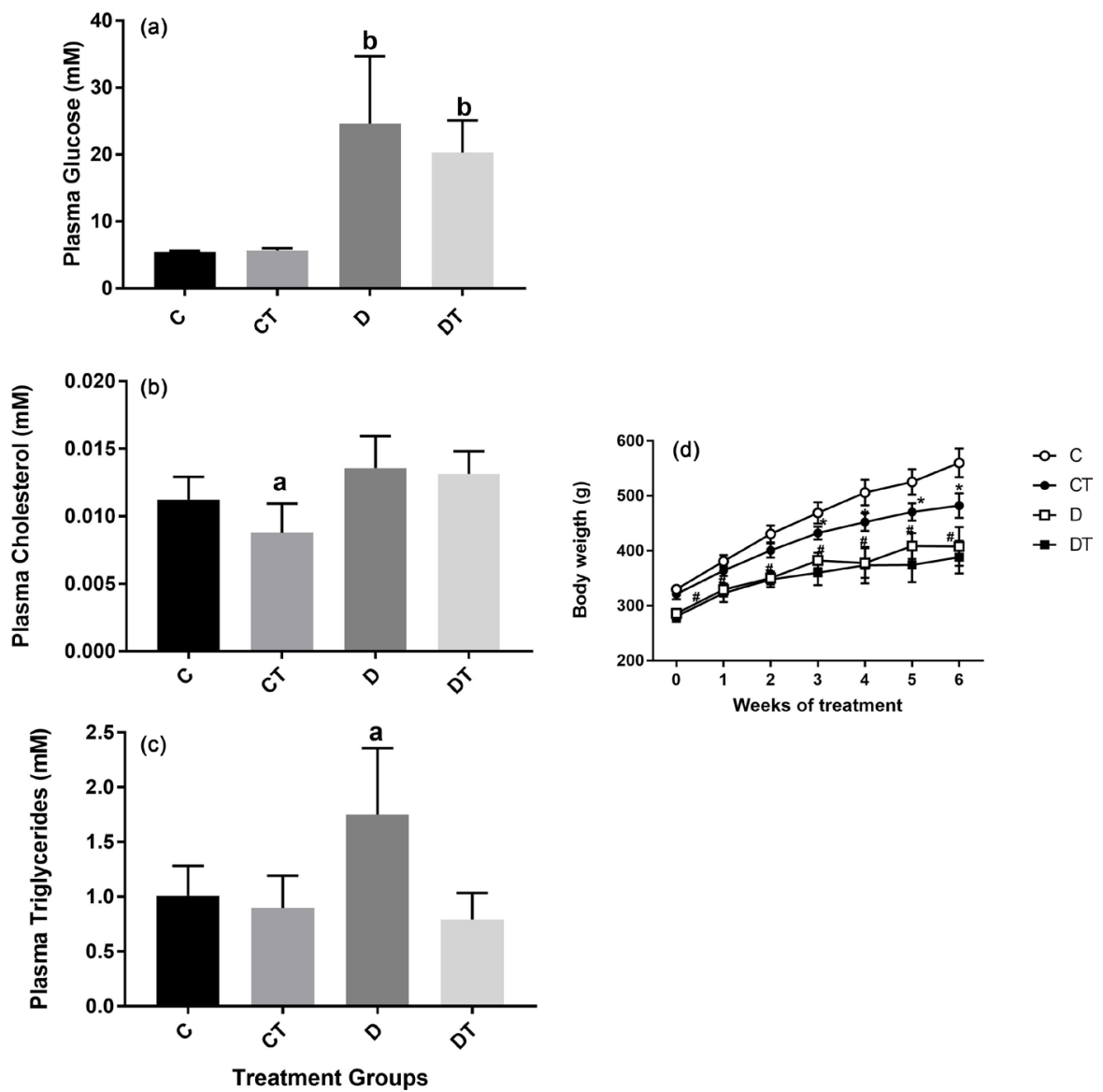

Figure 1. Effects of 6 weeks of treatment with SPI on plasma glucose, cholesterol, triglycerides levels and body weight in STZ-induced diabetic rats. The treatment groups were: control (C), control treated (CT), diabetic (D) and diabetic treated (DT). Data were expressed as mean \pm SEM and statistical analysis was performed using two-way ANOVA followed by Newman-Keuls multiple comparisons test, $\mathrm{p}<0.05$ taken as significant, $\mathrm{n}=$ 8 /group. adifferent from all other groups and bdifferent from control untreated and control treated groups.

\subsection{SPI $50 \mathrm{mg} / \mathrm{kg} /$ day Reduced Lipid Accumulation in the Liver of Treated Animals}

The control, control treated and diabetic treated groups showed normal histology with hepatocytes arranged in single-cell thick plates of liver tissue, which consists of a vast inter-anastomosing network of hepatocytes arranged in single-cell 
cords. The diabetic untreated group showed an intra-cytoplasmic accumulation of triglyceride. Fat droplets displaced the centrally located nucleus forming fat vacuoles that are well delineated and optically "empty". Many hepatocytes in the diabetic-untreated group possessed shrunken nuclei, ambiguous cell boundaries, granulated cytoplasm and dilated sinusoids (Figure 2).

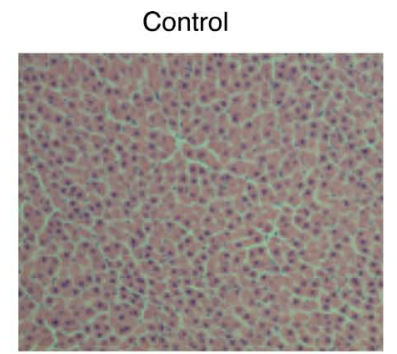

H\&E 200x magnification

Control

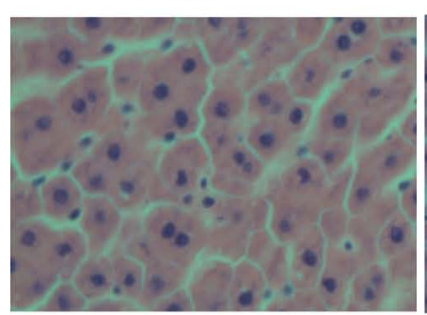

H\&E 400x magnification
Control T

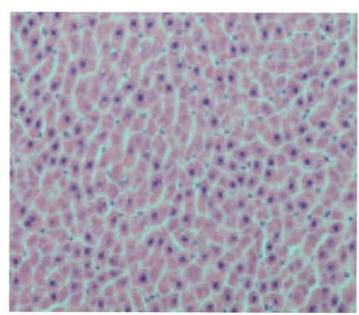

Control T

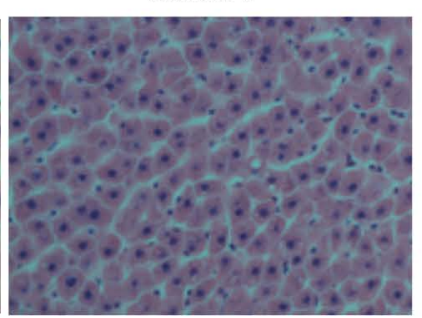

Diabetic

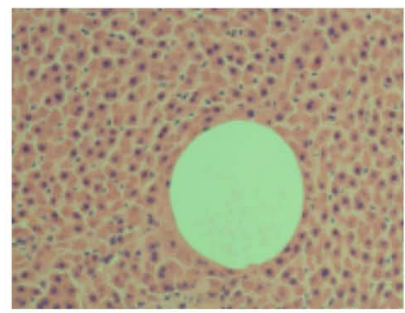

Diabetic

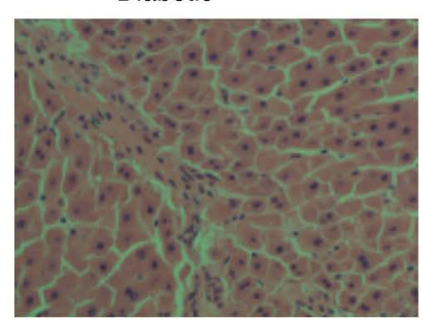

Diabetic T

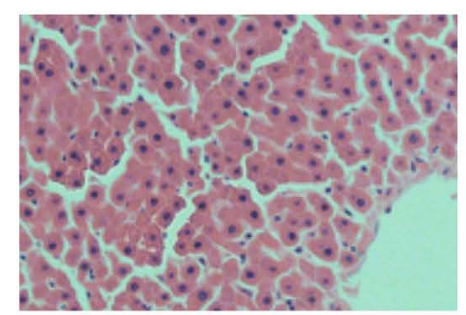

Diabetic $T$

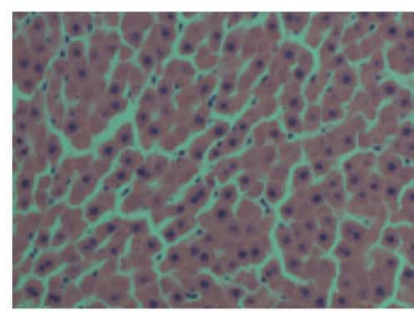

Figure 2. Effects of 6 weeks of treatment with SPI on lipid accumulation in STZ-induced diabetic rats. The 4 treatment groups were: control (C), control T (CT), diabetic (D) and diabetic T (DT). The control, control-treated and diabetic-treated groups showed normal histological features with hepatocytes arranged in single-cell thick plates. The diabetic-untreated group showed intra-cytoplasmic accumulation of triglyceride (neutral fats). Fat droplets displace the centrally located nucleus forming fat vacuoles.

\subsection{SPI $50 \mathrm{mg} / \mathrm{kg} /$ day Reduced Nitrite Levels in Diabetic Treated Rats}

Nitrite and total superoxide dismutase, 2 markers of oxidative stress, were measured in the tissues of the liver, brain and heart. There was no effect of SPI treatment on total superoxide dismutase. Treatment of diabetic rats with SPI 50 $\mathrm{mg} / \mathrm{kg} /$ day restored nitrite levels to control levels in liver tissue and significantly attenuated the increased nitrite content in both the heart and the brain (Figure 3 ).

\subsection{SPI $50 \mathrm{mg} / \mathrm{kg} /$ day Inhibited Lipid Peroxidation in Diabetic Treated Rats}

Malondialdehyde (MDA) levels, a marker of lipid peroxidation, were greatly increased in STZ-induced diabetic rats as compared to normal rats in liver, brain and heart tissue. The treatment of STZ-induced diabetic rats with SPI for 6 weeks restored whole-tissue MDA levels to normal in the liver and heart. In brain tissue MDA levels were not improved with SPI treatment as compared to the diabetic treated group (Figure 4). 

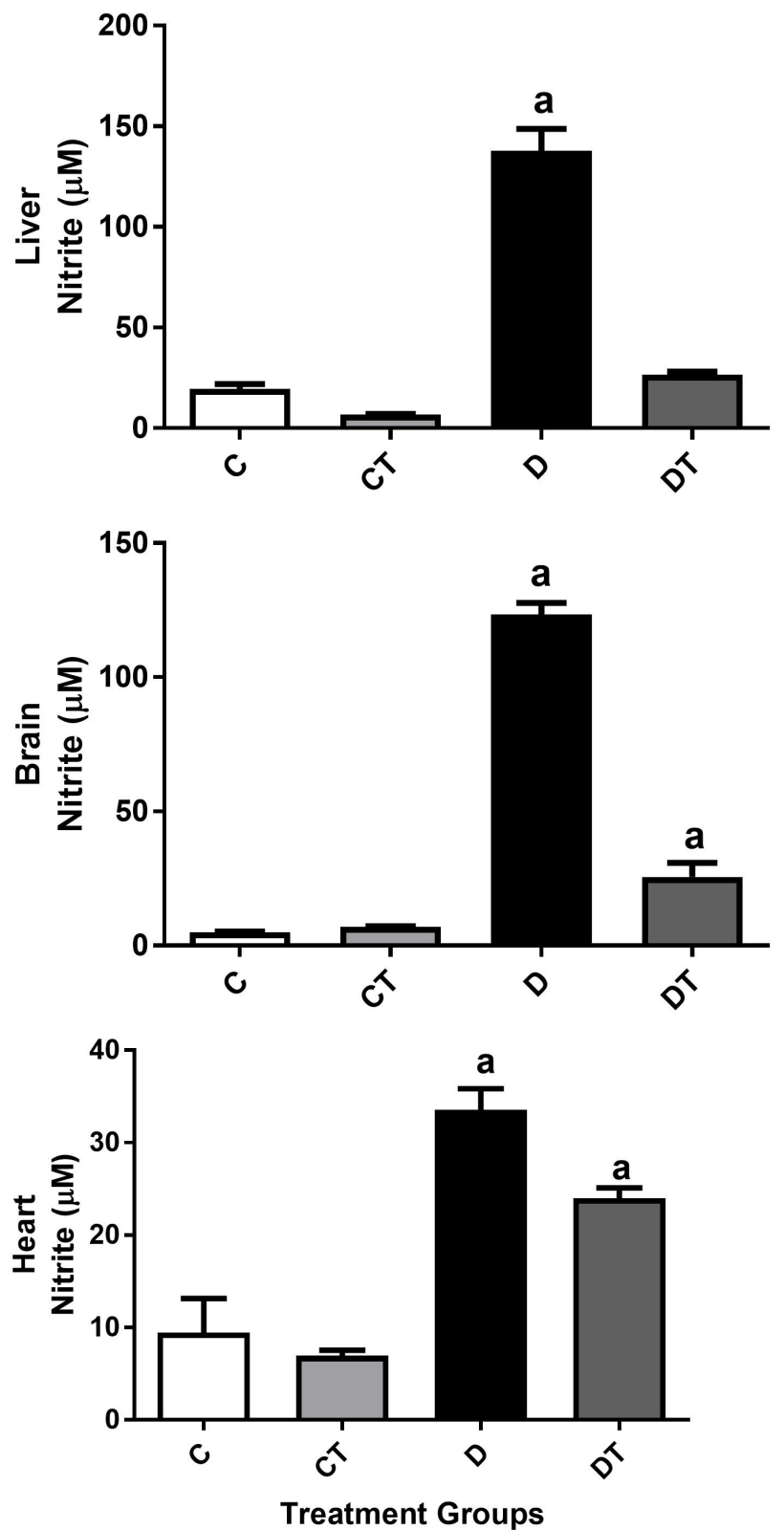

Figure 3. Effects of 6 weeks of treatment with SPI on nitrite content in the liver, brain and heart of STZ-induced diabetic rats. The treatment groups were: control (C), control treated (CT), diabetic (D) and diabetic treated (DT). Data were expressed as mean \pm SEM and statistical analysis was performed using two-way ANOVA followed by Newman-Keuls multiple comparisons test, $\mathrm{p}<0.05$ taken as significant, $\mathrm{n}=8$ /group. ${ }^{\text {adifferent }}$ from all other groups.

\subsection{SPI $50 \mathrm{mg} / \mathrm{kg} /$ day Produced an Improvement in the Cardiac Function in Control Treated Rats}

The treatment with SPI $50 \mathrm{mg} / \mathrm{kg} /$ day for 6 weeks did not restore the ability of the diabetic animals to respond to increases in filling pressure on all functional parameters studied. However SPI, at this same concentration, produced an improvement in cardiac parameters of $+\mathrm{dP} / \mathrm{dT}$ and $-\mathrm{dP} / \mathrm{dT}$ in control treated rats (Figure 5). 

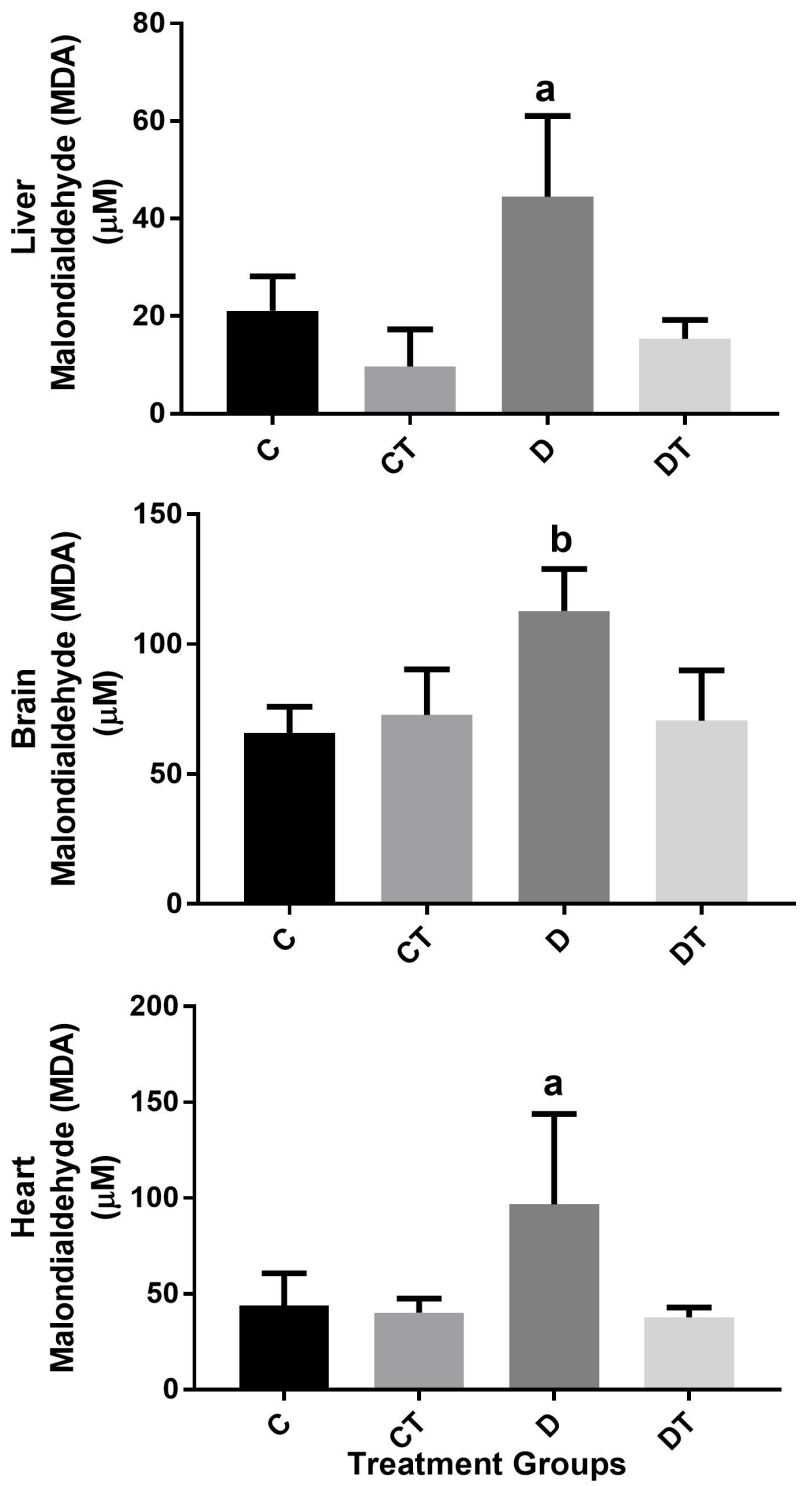

Figure 4. Effects of 6 weeks of treatment with SPI on malondialdehyde (MDA) content in the liver, brain and heart of STZ-induced diabetic rats. The treatment groups were: control (C), control treated (CT), diabetic (D) and diabetic treated (DT). Data were expressed as mean \pm SEM and statistical analysis was performed using two-way ANOVA followed by Newman-Keuls multiple comparisons test, $\mathrm{p}<0.05$ taken as significant, $\mathrm{n}=8$ /group a different from all other groups and $\mathrm{b}$ different from control group.

\section{Discussion}

Currently the hypoglycemic and hypolipidemic effects of SPI are well recognized through several in vivo studies carried out in rats and mice under various experimental conditions. Previous studies from our group in Brazil showed that the oral administration of SPI for 5 or 10 days, at the doses of 25,50 and 100 $\mathrm{mg} / \mathrm{kg} /$ day, significantly reduced glucose, triglyceride and cholesterol levels in alloxan induced diabetic rats [5]. Other groups have shown that SPI exhibited hypolipidemic and hypoglycemic activities through the lowering of blood glucose levels, regulating cholesterol and triglyceride and improving insulin resistance in 


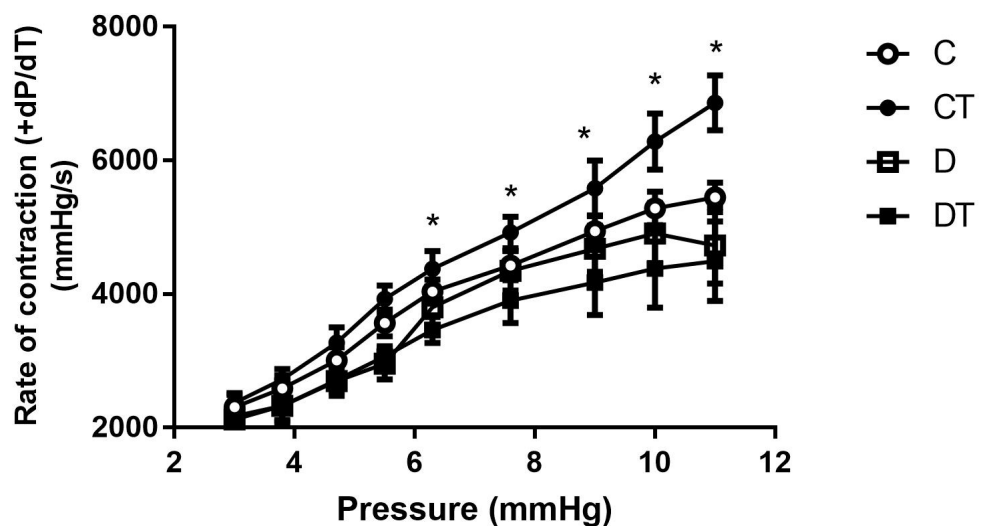

(a)

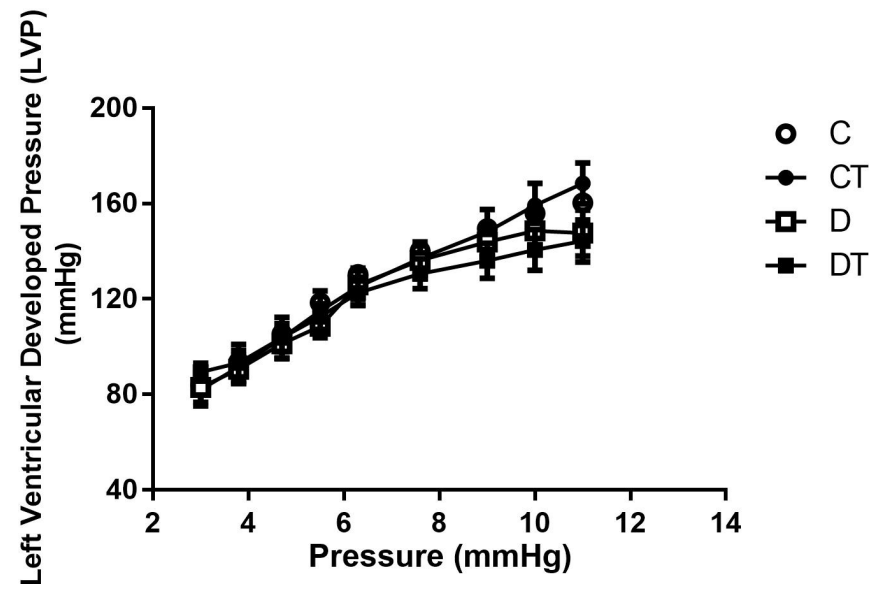

(b)

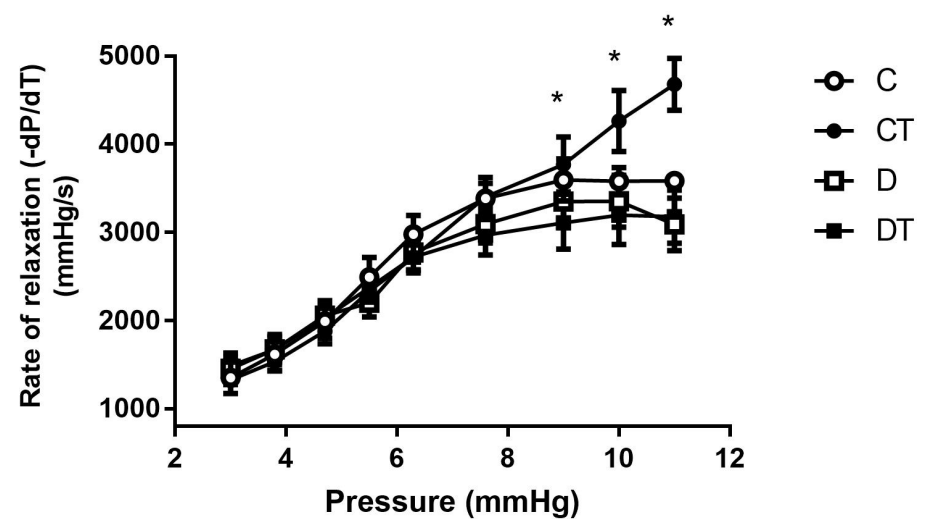

(c)

Figure 5. Effects of 6 weeks of treatment with SPI on rate of contraction $(+\mathrm{dP} / \mathrm{dT})$, on rate of left ventricular developed pressure (LVP) and on rate of relaxation $(-\mathrm{dP} / \mathrm{dT})$ at different left atrial filling pressures in STZ-induced diabetic rats. The treatment groups were: control $(C, n=5)$, control treated $(C T, n=7)$, diabetic $(D, n=5)$ and diabetic treated (DT, $n=5$ ). Data were expressed as mean \pm SEM and statistical analysis was performed using GLM repeated measures ANOVA followed by Newman-Keuls multiple comparisons test, $\mathrm{p}<0.05$ taken as significant, $\mathrm{n}=8$ /group ${ }^{\star}$ different from all other groups.

DM patients [21] [22] [23] [24] [25]. These findings suggest that SPI is a promising therapeutic agent for the management of diabetes. 
SPI presents cardiovascular benefits which primarily result from its hypolipidemic, antioxidant and anti-inflammatory activities, as demonstrated in a large number of preclinical studies and clinical trials [21]. In this experiment the intermidate dose of SPI $50 \mathrm{mg} / \mathrm{kg} /$ day was used (based on preliminary studies done by our partner group in Brazil [5]) to study the effect of SPI on oxidative stress, glycemic and lipid profile in the STZ diabetic animal model. However after 6 weeks of treatment the diabetic treated animals did not show a significant reduction in glycemic levels, perhaps which may be because the glycemic levels achieved in the STZ animals were highly elevated in this study. We hypothesized that the dose of SPI $50 \mathrm{mg} / \mathrm{kg} /$ day may have been too low in this animal model to reduce glycemic levels. However we did observe that SPI at this same dose significantly decreased triglyceride in diabetic treated animals and reduced plasma cholesterol levels in control treated animals.

Several animal and human studies have repeatedly reported the lipid-lowering effects of SPI [21] [22] [23] [24] [25]. However, the mechanisms of action of SPI on lipid metabolism are not well understood. Nagakoa and collaborators found that a concentrate of SPI inhibited jejunal cholesterol absorption and ileal bile acid reabsorption, proposing that $\mathrm{C}$-phycocyanin is the molecule responsible for this effect [26]. Furthermore, it was found that phycocyanin inhibits pancreatic lipase [27]. These effects could explain the hypocholesterolemic and hypotriacylglycerolemic effects of SPI [28].

We have also shown in this study that SPI $50 \mathrm{mg} / \mathrm{kg} /$ day (6 weeks) significantly reduced the weight of the control treated animals when compared to control, while SPI did not change the weight of diabetic treated animals when compared to the diabetic control. Literature results of studies with SPI at higher does have shown that SPI succeeded inducing either an improvement in body weight or no weight reduction in human and animals [21]. However, more recent studies have demonstrated that Spirulina platensis supplementation was effective in weight regulation, serum total cholesterol and appetite reduction in overweight patients [29] [30].

In this study we showed a normal morphological arrangement of the hepatocytes in SPI treated diabetic animals as compared to hepatocytes from untreated diabetic animals in which fat droplets displaced the centrally located nucleus forming fat vacuoles. The observed fatty degeneration is linked to insulin deficiency and the dysregulation of mitochondrial $\beta$-oxidation of fatty acids. This leads to the esterification of fatty acids to triglyceride in the cytoplasm, which is characterized by the presence of multiple triglyceride droplets within the hepatocytes [31].

Many studies have shown that SPI has antioxidant properties [5] [21] [24] [32] [33] [44] [45]. These findings suggest that SPI can prevent oxidative stress and improve the antioxidant status. In the present study we measured some markers of oxidative stress in liver, heart and brain homogenate to examine whether SPI could affect the oxidative state. 
The concentration of nitric oxide (NO) (estimated as concentration of nitrite) was increased in all tissues of diabetic rats. NO levels were brought back to near normal values after the treatment with SPI $50 \mathrm{mg} / \mathrm{kg} /$ day. Generally, NO at physiological levels produces many benefits to the vascular system. However, increased oxidative stress and subsequent activation of the transcription factor NF- $\beta$ enhanced the NO production, which is believed to be a mediator of damage to beta-cells [34].

The reduction of nitrite production in different tissues such as brain, heart and liver in our study could be considered an important factor of protection against oxidative damage to DNA, indicating its important protective effect against STZ-induced damage and these results are corroborated by numerous researchers who have studied the antioxidant and anti-inflammatory properties of SPI [35] [36] [37]. Many of the studies investigating the antioxidant effect of SPI have attributed this property to phycocyanin since this phycobilin pigment has radical scavenging properties, noting that some of these reports have also shown that phycocyanin directly inhibits $\mathrm{NAD}(\mathrm{P}) \mathrm{H}$ oxidase activity [38] [39] and that $\mathrm{NAD}(\mathrm{P}) \mathrm{H}$ oxidases may be the main source of ROS in the tissues of diabetic animals and patients [40] [41].

In addition, the level of malondialdehyde (MDA) was analyzed as a lipid peroxidation marker since it has an important role in the pathogenesis and the complications of diabetes. The results of the present analysis clearly highlight the efficacy of SPI as an MDA lowering agent. The MDA levels in diabetic rats treated with SPI were significantly lower in comparison to the diabetic control. $(\mathrm{P}<0.05)$.

In this study we also evaluated the antioxidant biomarker enzyme superoxide dismutase (SOD), however there were no differences in total SOD activity among the groups studied.

Other researchers have tried to correlate the elevated lipid profile in diabetes to the development of cardiac dysfunction [19] [20] [42]. Lipid abnormalities associated with diabetes may lead to alterations in myocardial enzyme systems, subcellular organelles and myocardial fuel supply and eventually to cardiac disease [19].

In this study hearts from control and diabetic rats were isolated and cardiac performance was evaluated under physiological and superphysiological filling pressures, simulated by increases in left atrial filling pressure from 2.5 to 11 mmHg. Paralleling the elevation of plasma glucose and triglycerides, the untreated diabetic group exhibited decreased cardiac performance as assessed by their inability to respond to increases in left atrial filling pressure. Treatment with SPI for 6 weeks did not restore the ability of the treated diabetic animals to respond to increases in filling pressure on the functional parameters studied. However, SPI in this same concentration produced an improvement in cardiac hemodynamic parameters such as $\mathrm{dP} / \mathrm{dTm} \max$ and $\mathrm{dP} / \mathrm{dTmin}$ in control rats, suggesting a possible positive inotropic effect of Spirulina.

The ineffectiveness of SPI in blocking the effects of systolic and diastolic dys- 
function in diabetic rats may be due to the low dose used in this experiment, as some researchers reported evidence that both SPI and C-phycocyanin can have a preventive effect on drug-induced cardiac side effects as well as a protective effect during heart attacks [43] [44] [45]. In the first two studies, they tested first SPI and then C-phycocyanin as protectants against the adverse cardiac side effects of doxorubicin, a chemotherapy drug. They concluded that both SPI and C-phycocyanin significantly protected the mice from the cardiotoxic effects and was referred to as a "crucial role of the antioxidant nature of SPI and C-phycocyanin in cardioprotection" [44] [45]. Another study from the same group demonstrated that pre-ischemic infusions of C-phycocyanin and SPI were cardioprotective against ischemia-reperfusion (I/R) injury, leading to enhanced recovery of contractile function, attenuation of infarct size, decreased apoptosis, and suppression of oxidative stress in the post-ischemic reperfused heart. These results suggest that the underlying signaling mechanism(s) involved C-phycocyanin attenuation of I/R-induced cardiac dysfunction through its antioxidant and antiapoptotic actions and modulation of p38 MAPK and ERK1/2 [45].

\section{Conclusion}

Further studies will be needed to confirm an effect of SPI on diabetic cardiomyopathy. However this study has shown that SPI significantly suppressed nitrite generation and lipoperoxidation in the hearts of animals diabetics, as well as an improvement in the cardiac function SPI-treated control rats which is consistent with several studies that demonstrated that ROS produced in diabetic myocardial tissues causes oxidative stress-mediated injury that is protected by antioxidants.

\section{Acknowledgements}

The authors are thankful to the Science without Borders Project, CNPq (the Brazilian National Council for Scientific and Technological Development) for the financial support of this study.

\section{References}

[1] Tietze, H.W. (2004) Spirulina Micro Food Macro Blessing. 4th Edition, Harald W. Tietze Publishing, Australia.

[2] Kay, R.A (1991) Microalgae as Food and Supplement. Critical Reviews in Food Science and Nutrition, 30, 555-573. https://doi.org/10.1080/10408399109527556

[3] Piñero Estrada, J.E., Bermejo Bescós, P. and Villar del Fresno, A.M. (2001) Antioxidant Activity of Different Fractions of Spirulina Protean Extract. Farmaco, 56, 497-500. https://doi.org/10.1016/S0014-827X(01)01084-9

[4] Chen, J.C., Liu, K.S., Yang, T.J., Hwang, J.H., Chan, Y.C. and Lee, I.T. (2012) Spirulina and C-Phycocyanin Reduce Cytotoxicity and Inflammation-Related Genes Expression of Microglial Cells. Nutritional Neuroscience, 15, 252-256. https://doi.org/10.1179/1476830512Y.0000000020

[5] Joventino, I.P., Alves, H.G., Neves. L.C., Pinheiro-Joventino, F., Leal, L.K., Neves, 
S.A., et al. (2012) The Microalga SPI Presents Anti-Inflammatory Action as Well as Hypoglycemic and Hypolipidemic Properties in Diabetic Rats. Journal of Complementary and Integrative Medicine, 9, 1553-3840.

https://doi.org/10.1515/1553-3840.1534

[6] Lima, F.A.V., Joventino, I.P., Joventino, F.P., de Almeida A.C., Neves. K.R.T., do Carmo, M.R., et al. (2017) Neuroprotective Activities of Spirulina platensis in the 6-OHDA Model of Parkinson's Disease Are Related to Its Anti-Inflammatory Effects. Neurochemical Research, 42, 3390-3400.

https://doi.org/10.1007/s11064-017-2379-5

[7] Pentón-Rol, G., Martínez-Sánchez, G., Cervantes-Llanos, M., Lagumersindez-Denis, N., Acosta-Medina, E.F., Falcón-Cama, V., et al. (2011) C-Phycocyanin Ameliorates Experimental Autoimmune Encephalomyelitis and Induces Regulatory T Cells. International Immunopharmacology, 11, 29-38.

https://doi.org/10.1016/j.intimp.2010.10.001

[8] Lee, J., Park, A., Kim, M.J., Lim, H.J., Rha, Y.A. and Kang, H.G. (2017) Spirulina Extract Enhanced a Protective Effect in Type 1 Diabetes by Anti-Apoptosis and Anti-ROS Production. Nutrients, 9, E1363. https://doi.org/10.3390/nu9121363

[9] Farouk, K., El-Baz, F., Hanan Aly., El-Sayed A.B. and Amal, A. (2013) Mohamed. Role of Spirulina platensis in the Control of Glycemia in DM2 Rats. International Journal of Scientific \& Engineering Research, 4, 1731-1740.

[10] American Diabetes Association (2012) Diagnosis and Classification of Diabetes Mellitus. Diabetes Care, 35, 564-571.

[11] Bugger, H. and Abel, E.D. (2014) Molecular Mechanisms of Diabetic Cardiomyopathy. Diabetologia, 57, 660-671. https://doi.org/10.1007/s00125-014-3171-6

[12] Secrest, A.M., Becker, D.J., Kelsey, S.F., LaPorte, R.E. and Orchard, T.J. (2010) All-Cause Mortality Trends in a Large Population-Based Cohort with Long-Standing Childhood-Onset Type 1 Diabetes: The Allegheny County Type 1 Diabetes Registry. Diabetes Care, 33, 2573-2579. https://doi.org/10.2337/dc10-1170

[13] Dobrin, J.S. and Lebeche, D. (2010) Diabetic Cardiomyopathy: Signaling Defects and Therapeutic Approaches. Expert Review of Cardiovascular Therapy, 8, 373-391. https://doi.org/10.1586/erc.10.17

[14] Cai, H. and Harrison, D.G. (2000) Endothelial Dysfunction in Cardiovascular Diseases: The Role of Oxidant Stress. Circulation Research, 87, 840-844. https://doi.org/10.1161/01.RES.87.10.840

[15] Guzik T.J., Mussa, S., Gastaldi, D., Sadowski, J., Ratnatunga, C., Pillai, R. and Channon, K.M. (2002) Mechanisms of Increased Vascular Superoxide Production in Human Diabetes Mellitus: Role of $\mathrm{NAD}(\mathrm{P}) \mathrm{H}$ Oxidase and Endothelial Nitric Oxide Synthase. Circulation, 105, 1656-1662. https://doi.org/10.1161/01.CIR.0000012748.58444.08

[16] Suzuki, H., Kayama, Y., Sakamoto, M., Iuchi, H., Shimizu, I., Yoshino, T., et al. (2015) Arachidonate 12/15-Lipoxygenase-Induced Inflammation and Oxidative Stress Are Involved in the Development of Diabetic Cardiomyopathy. Diabetes, 64, 618-630. https://doi.org/10.2337/db13-1896

[17] Kayama, Y., Raaz, U., Jagger, A., Adam, M., Schellinger, I.N, Sakamoto, M., et al. (2015) Diabetic Cardiovascular Disease Induced by Oxidative Stress. International Journal of Molecular Sciences, 16, 25234-25263. https://doi.org/10.3390/ijms161025234

[18] Zarrouk, C. (1996) Contribution to the Study of a Cyanophycea: Influence of Various Physical and Chemical Factors on the Growth and Photosynthesis of Spirulina 
Maxima. PhD Thesis, Fac. Sci., University of Paris, Paris.

[19] Rodrigues, B., Xiang, H. and McNeill, J.H. (1988) Effect of Lcarnitine Treatment on Lipid Metabolism and Cardiac Performance in Chronically Diabetic Rats. Diabetes, 37, 1358-1364. https://doi.org/10.2337/diab.37.10.1358

[20] Rodrigues, B., Goyal, R.K. and McNeill, J.H. (1986) Effects of Hydralazine on STZ-Induced Diabetes Rats-Prevention of Hyperlipidemia and Improvement in Cardiac Function. The Journal of Pharmacology and Experimental Therapeutics, 237, 292-299.

[21] Deng, R. and Chow, T.J. (2010) Hypolipidemic, Antioxidant, and Antiinflammatory Activities of Microalgae Spirulina. Cardiovascular Therapeutics, 28, 33-45. https://doi.org/10.1111/j.1755-5922.2010.00200.x

[22] Lee, E.H., Park, J.E., Choi, Y.J., Huh, K.B. and Kim, W.Y. (2008) A Randomized Study to Establish the Effects of Spirulina in Type 2 Diabetes Mellitus Patients. $\mathrm{Nu}$ trition Research and Practice, 2, 295-300. https://doi.org/10.4162/nrp.2008.2.4.295

[23] Mani, U.V., Desai, U.V.S. and Iyer, U. (2000) Studies on the Long-Term Effect of Spirulina Supplementation on Serum Lipid Profile and Glycated Proteins in NIDDM Patients. Journal of Nutraceuticals, Functional and Medical Foods, 2, 25-32. https://doi.org/10.1300/J133v02n03_03

[24] Park, J.Y. and Kim, W.Y. (2003) The Effect of Spirulina on Lipid Metabolism, Antioxidant Capacity and Immune Function in Korean Elderly. The Korean Journal of Nutrition, 36, 287-297.

[25] Parikh, P., Mani, U. and Iyer, U. (2001) Role of Spirulina in the Control of Glycemia and Lipidemia in Type 2 Diabetes Mellitus. Journal of Medicinal Food, 4, 193-199. https://doi.org/10.1089/10966200152744463

[26] Nagaoka, S., Shimizu, K., Kaneko, H., Shibayama, F., Morikawa, K., Kanamaru, Y., et al. (2005) A Novel Protein C-Phycocyanin Plays a Crucial Role in the Hypocholesterolemic Action of Spirulina platensis Concentrate in Rats. Journal of Nutrition, 135, 2425-2430. https://doi.org/10.1093/jn/135.10.2425

[27] Li-Kun, H., Dong-Xia, L., Lan, X., Xiao-Jie, G., Yasumasa, K., Isao, S. and Hiromichi, O. (2006) Isolation of Pancreatic Lipase Activity-Inhibitory Component of Spirulina platensis and It Reduce Postprandial Triacylglycerolemia. Yakugaku Zasshi, 126, 43-49. https://doi.org/10.1248/yakushi.126.43

[28] Iwata, K., Inayama, T. and Kato, T. (1990) Effects of Spirulina platensis on Plasma Lipoprotein Lipase Activity in Fructose-Induced Hyperlipidemic Rats. Journal of Nutritional Science and Vitaminology, 36, 165-171. https://doi.org/10.3177/jnsv.36.165

[29] Miczke, A.A., SzulInska, M., Hansdorfer-Korzon, R., Kregielsk-Narozna, M., Suliburska, J., Walkowiak, J., et al. (2016) Effects of Spirulina Consumption on Body Weight, Blood Pressure, and Endothelial Function in Overweight Hypertensive Caucasians: A Double Blind, Placebo-Controlled, Randomized Trial. European Review for Medical and Pharmacological Sciences, 20, 150-156.

[30] Zeinalian, R., Farhangi, M.A., Shariat, A. and Saghafi-Asl, M. (2017) The Effects of Spirulina Platensis on Anthropometric Indices, Appetite, Lipid Profile and Serum Vascular Endothelial Growth Factor (VEGF) in Obese Individuals: A Randomized Double Blinded Placebo Controlled Trial. BMC Complementary and Alternative Medicine, 17, 225. https://doi.org/10.1186/s12906-017-1670-y

[31] Fromenty, B. and Pessayre, D. (1995) Inhibition of Mitochondrial Beta-Oxidation as a Mechanism of Hepatotoxicity. Pharmacology \& Therapeutics, 67, 101-154. https://doi.org/10.1016/0163-7258(95)00012-6 
[32] Bertolin, T.E., Pilatti, D., Giacomini, A.C.V.V., Bavaresco, C.S., Colla, L.M. and Costa, J.A.V. (2009) Effect of Microalga Spirulina platensis (Arthrospira platensis) on Hippocampus Lipoperoxidation and Lipid Profile in Rats with Induced Hypercholesterolemia. Brazilian Archives of Biology and Technology, 52, 1253-1259. https://doi.org/10.1590/S1516-89132009000500024

[33] Dartsch, P.C. (2008) Antioxidant Potential of Selected Spirulina platensis Preparations. Phytotherapy Research, 22, 627-633. https://doi.org/10.1002/ptr.2310

[34] Malik, Z.A., Tabassum, N. and Sharma, P.L. (2013) Attenuation of Experimentally Induced Diabetic Neuropathy in Association with Reduced Oxidative-Nitrosative Stress by Chronic Administration of Momordica charantia. Advances in Bioscience and Biotechnology, 4, 356-363. https://doi.org/10.4236/abb.2013.43047

[35] Lee, J., Park, A., Kim, M.J., Lim, H.J., Rha, Y.A. and Kang, H.G. (2017) Spirulina Extract Enhanced a Protective Effect in Type 1 Diabetes by Anti-Apoptosis and Anti-ROS Production. Nutrients, 9, 1363. https://doi.org/10.3390/nu9121363

[36] Romay, C., Delgado, R., Remirez, D., González, R. and Rojas, A. (2001) Effects of Phycocyanin Extract on Tumor Necrosis Factor-Alpha and Nitrite Levels in Serum of Mice Treated with Endotoxin. Arzneimittelforschung, 51, 733-736.

[37] Romay, C., González, R., Ledón, N., Remirez, D. and Rimbau, V. (2003) C-Phycocyanin: A Biliprotein with Antioxidant, Anti-Inflammatory and Neuroprotective Effects. Current Protein \& Peptide Science, 4, 207-216. https://doi.org/10.2174/1389203033487216

[38] Riss, J., Décordé, K., Sutra, T., Delage, M., Baccou, J.C., Jouy, N., et al. (2007) Phycobiliprotein C-Phycocyanin from Spirulina platensis Is Powerfully Responsible for Reducing Oxidative Stress and NADPH Oxidase Expression Induced by an Atherogenic Diet in Hamsters. Journal of Agricultural and Food Chemistry, 55, 7962-7967. https://doi.org/10.1021/jf070529g

[39] Lanone, S., Bloc, S., Foresti, R., Almolki, A., Taillé, C., Callebert, J., et al. (2005) Bilirubin Decreases nos2 Expression via Inhibition of NAD(P)H Oxidase: Implication for Protection against Endotoxic Shock in Rats. FASEB Journal, 19, 1890-1892. https://doi.org/10.1096/fj.04-2368fje

[40] Inoguchi, T., Li, P., Umeda, F., Yu, H.Y., Kakimoto, M., Imamura, M., et al. (2000) High Glucose Level and Free Fatty Acid Stimulate Reactive Oxygen Species Production through Protein Kinase C-Dependent Activation of NAD(P)H Oxidase in Cultured Vascular Cells. Diabetes, 49, 1939-1945.

https://doi.org/10.2337/diabetes.49.11.1939

[41] Inoguchi, T. and Nawata, H. (2005) NAD(P)H Oxidase Activation: A Potential Target Mechanism for Diabetic Vascular Complications, Progressive Beta-Cell Dysfunction and Metabolic Syndrome. Current Drug Targets, 6, 495-501.

https://doi.org/10.2174/1389450054021927

[42] Bayeva, M., Sawicki, K.T. and Ardehali, H. (2013) Taking Diabetes to Heart-Deregulation of Myocardial Lipid Metabolism in Diabetic Cardiomyopathy. Journal of the American Heart Association, 2, e000433. https://doi.org/10.1161/JAHA.113.000433

[43] Khan, M., Varadharaj, S., Ganesan, L.P., Shobha, J.C. and Naidu, M.U. (2005) C-Phycocyanin Protects against Ischemia-Reperfusion Injury of Heart through Involvement of p38 MAPK and ERK Signaling. American Journal of Physiology. Heart and Circulatory Physiology, 290, 2136-2145. https://doi.org/10.1152/ajpheart.01072.2005

[44] Khan, M., Shobha, J.C., Mohan, I.K., Naidu, M.U., Sundaram, C., Singh, S., et al. 
(2005) Protective Effect of SPI against Doxorubicin-Induced Cardiotoxicity. Phytotherapy Research, 19, 1030-1037. https://doi.org/10.1002/ptr.1783

[45] Khan, M., Varadharaj, S., Shobba, J.C., Naidu, M.U., Parinandi, N.L., Kutala, V.K., et al. (2006) C-Phycocyanin Ameliorates Doxorubicin-Induced Oxidative Stress and Apoptosis in Adult Rat Cardiomyocytes. Journal of Cardiovascular Pharmacology, 47, 9-20. https://doi.org/10.1097/01.fjc.0000191520.48404.27 